\title{
Thalidomide combined with chemo-radiotherapy for treating esophageal cancer: A randomized controlled study
}

\author{
JIAN WANG ${ }^{1}$, JINGPING YU ${ }^{2}$, JIANLIN WANG ${ }^{2}$, XINCHU NI $^{2}$, \\ ZHIQIANG SUN ${ }^{2}$, WEI SUN ${ }^{2}$, SUPING SUN ${ }^{2,3}$ and YUTING LU ${ }^{2}$ \\ ${ }^{1}$ Department of Radiotherapy, Jiangyin People's Hospital, Jiangyin, Jiangsu 214400; ${ }^{2}$ Department of Radiotherapy, \\ The Affiliated Changzhou No. 2 People's Hospital, Nanjing Medical University, Changzhou, Jiangsu 213003; \\ ${ }^{3}$ Department of Radiotherapy, Xuzhou No. 3 People's Hospital, Xuzhou, Jiangsu 221000, P.R. China
}

Received February 13, 2018; Accepted March 18, 2019

DOI: $10.3892 / 01.2019 .10354$

\begin{abstract}
The aim of the present study was to investigate the efficiency and safety of a combination of thalidomide and chemo-radiotherapy (CRT) for treating esophageal cancer (EC). Eligible patients received two cycles of chemotherapy using paclitaxel liposome and cisplatin concurrently with three-dimensional radiotherapy. Following radiotherapy, two cycles of maintenance chemotherapy were performed. Patients with elevation of vascular endothelial growth factor (VEGF) during radiotherapy were randomly divided into: i) a test group $(n=31)$, who received a combination of CRT and thalidomide; and ii) a control group $(n=30)$, who received CRT only. Patients with locally advanced EC in the test group demonstrated a significantly improved 3-year overall survival (OS) rate, progression-free survival (PFS) rate, local control and median PFS time compared with the control group $(\mathrm{P}<0.05)$. Multivariate analysis indicated that Tumor-Node-Metastasis (TNM) stage was associated with the OS time, while TNM stage and the residence of cancer cells following radiotherapy were associated with PFS time. The present data indicate that thalidomide contributes to an
\end{abstract}

Correspondence to: Professor Yuting Lu, Department of Radiotherapy, The Affiliated Changzhou No. 2 People's Hospital, Nanjing Medical University, 29 Xinglong Lane, Tianning, Changzhou, Jiangsu 213003, P.R. China

E-mail: lyt513519@qq.com

Professor Suping Sun, Department of Radiotherapy, Xuzhou No. 3 People's Hospital, 131 Huancheng Road, Xuzhou, Jiangsu 221000, P.R. China

E-mail: ssp56@126.com

Abbreviations: EC, esophageal cancer; CRT, chemo-radiotherapy; VEGF, vascular endothelial growth factor; OS, overall survival; PFS, progression-free survival; LC, local control; CR, complete response; PR, partial response

Key words: esophageal cancer, chemo-radiotherapy, thalidomide, vascular endothelial growth factor, prognosis improvement of prognosis for patients with locally advanced EC with elevated serum VEGF levels during radiotherapy. In addition, the toxicities induced by thalidomide were demonstrated to be tolerable.

\section{Introduction}

Esophageal cancer (EC), a common malignancy of the digestive tract, is one of the leading causes for cancer-associated mortality worldwide (1). Surgery is not recommended for $\mathrm{EC}$ as the majority of patients $(60 \%)$ present with advanced EC upon diagnosis (2). Therefore, radiotherapy-based combined modality is considered a major treatment option for EC. Unfortunately, a large percentage of patients with EC (60-70\%) exhibit a poor response following non-surgical treatment, including local recurrence and/or distal metastasis, with a 5-year survival rate of only $20-40 \%(3,4)$.

Thalidomide, initially used as a sedative, has been reported to be effective for the management of vomiting and nausea in pregnancy; however, its application is hindered as it can induce congenital disabilities in neonates (5). In 2006, thalidomide was approved by Food and Drug Administration for treating newly diagnosed multiple myeloma as it demonstrated antitumor activity in refractory multiple myeloma possibly by inhibiting angiogenesis (6). In addition, thalidomide has been considered as a treatment option for solid tumors due to its anti-angiogenesis effects (7). It has been demonstrated that EC cells exhibit increased sensitivity to a combination of radiotherapy and thalidomide (8-10). Our previous study revealed that a variation of serum vascular endothelial growth factor (VEGF) could predict the prognosis of patients with EC during chemo-radiotherapy (CRT). Additionally, the overall survival (OS) and progression-free survival (PFS) time in patients with elevated serum VEGF levels were significantly lower compared with patients with a decreased serum VEGF level (11). In a retrospective analysis, Yu et al (12) reported radiotherapy combined with thalidomide triggered a down-regulation of VEGF expression, but it caused no OS extension. The present study evaluated patients with EC with a poor prognosis, who exhibited elevated serum VEGF level during radiotherapy, with the aim of comparing the treatment efficiency, prognosis 
and side-effects between CRT and a combination of CRT and thalidomide.

\section{Materials and methods}

Clinical data. A total of 215 patients (male: 152; female: 63 , age: $40-87$ years, median age: $66.3 \pm 9.9$ years) diagnosed with esophageal squamous carcinoma, who were admitted to the Department of Radiotherapy, the Affiliated Changzhou No. 2 People's Hospital, Nanjing Medical University (Changzhou, China) between February 2011 and December 2015, were enrolled in the present study. The inclusion criteria were as follows: i) An Eastern Cooperative Oncology Group performance status score of 0 or 1 ; ii) received no treatment previously; iii) age $\leq 75$ years; iv) denied surgical treatment or with contraindications of surgery; v) lesion size $\leq 10 \mathrm{~cm}$; vi) absence of esophageal perforation; vii) no severe hepatic, renal and cardiopulmonary disease; viii) no severe cachexia; ix) Karnofsky performance score (KPS) (13) $\geq 80$; and iix) a survival time of $>3$ months from the time of diagnosis. Pregnant patients, patients who were breast-feeding and patients with any other malignancy were excluded from the study. VEGF elevation was defined as an elevation of serum VEGF of $\geq 16.2 \mathrm{ng} / \mathrm{l}$ during radiation compared with that before radiation. The value of $16.2 \mathrm{ng} / \mathrm{l}$ was defined according to the two-fold of the standard $(8.1 \mathrm{ng} / \mathrm{l})$. The number of patients with increased, stable or decreased VEGF during radiotherapy was 61,88 and 66 , respectively. Patients with elevated VEGF during radiotherapy $(n=61)$ were randomly divided into: i) a test group $(n=31)$, who received a combination of CRT and thalidomide; and ii) a control group $(n=30)$, who received CRT only. The criteria for the EC staging were based on the guidelines proposed by the 7 th edition of the American Joint Committee on Cancer Tumor-Node-Metastasis (TNM) staging system (14). Written informed consent was obtained from each patient. The study protocols were approved by the Ethics Committee of the Second People's Hospital of Changzhou Affiliated to Nanjing Medical University (Nanjing, China). This clinical trial was registered in the United States Trial Registry (ID: NCT01551641).

Treatment regimen. CT simulation was performed in all patients in the supine position. CT images were obtained at a 5-mm thickness throughout the entire neck and thorax. Treatment plans were generated with a three-dimensional planning system (ADAC-Pinnacle 3; Philips Medical Systems, Inc.; version 9.3). Irradiation was delivered with 6-MV photon energy through three-dimensional conformal radiotherapy or intensity modulated irradiation therapy. Gross tumor volume (GTV) was defined as any visible primary tumor on the CT or esophageal barium study, as well as metastatic lymph nodes. Metastatic nodes were identified based on the following radiographic criteria: Nodes $\geq 1 \mathrm{~cm}$ in the shortest axis in the intra-abdominal and/or intrathoracic regions and nodes beside the recurrent nerve with the shortest axis of $\geq 0.5 \mathrm{~cm}$. Clinical target volume (CTV) was defined as the GTV plus $3 \mathrm{~cm}$ of proximal and distal normal esophagus without lateral margins. Planning target volume was termed by adding a $1-\mathrm{cm}$ margin around the CTV. For radiotherapy, patients were treated 5 days per week (1.8-2.0 Gy) with a total dose of 50-70 Gy. The maximal dose for the spinal cord was $<45 \mathrm{~Gy}$ and the mean dose for the lung was $\leq 13 \mathrm{~Gy}$. The volume of the whole lung receiving $\geq 20 \mathrm{~Gy}$ and volume of the whole heart receiving $\geq 50$ Gy was $<28$ and $45 \%$, respectively. The regimen of chemotherapy consisted of paclitaxel (Lvye Pharma, www.luyesike.com, Nanjing, China; d1, $135 \mathrm{mg} / \mathrm{m}^{2}$ ) and cisplatin (Jiangsu Hanson Pharmaceutical Co., Ltd., Lianyungang, China; d2-5, $20 \mathrm{mg} / \mathrm{m}^{2}$ ). Two cycles of chemotherapy were performed concurrently with the radiotherapy. Following radiotherapy, two cycles (21-28 days for each cycle) of maintenance chemotherapy were performed. In the test group, the patients received thalidomide with an initial dose of $200 \mathrm{mg} / \mathrm{day}$ and then the dose was increased to $300 \mathrm{mg} /$ day in the absence of moderate or severe side-effects within 1 week.

Determination of VEGF. Peripheral blood $(2 \mathrm{ml})$ was collected before, during weeks 2-4 of radiotherapy and 1 week after radiotherapy. Blood samples were centrifuged at $999 \mathrm{x} g$ at $4^{\circ} \mathrm{C}$ for $10 \mathrm{~min}$ and the serum was stored at $-70^{\circ} \mathrm{C}$ for further analyses. The level of VEGF was determined using a commercial ELISA kit for VEGF (cat. no., 69-50044; http://www. mskbio.com/search.aspx?word=VEGF; International Capital Corporation Limited, Beijing, China), according to the manufacturer's protocol.

Evaluation of treatment efficiency and side-effects. The efficiency was evaluated by a barium esophagogram and thoracic computed tomography (CT) scan 1 month after radiotherapy. The primary lesions in the esophagus were evaluated by barium esophagogram. Treatment efficiency of the lymph node metastasizing lesions was evaluated based on the response evaluation criteria in solid tumors (2.1) guidelines (15). The side-effects of radiotherapy were evaluated using the guidelines proposed by the Radiation Therapy Oncology Group (16). The side-effects of chemotherapy and thalidomide were evaluated using the toxicity criteria by the World Health Organization (12). Hemoglobin: Grade $0 \geq 11.0 \mathrm{~g} / \mathrm{l}$; grade 1, 9.5-11.0 g/l; grade 2, 7.5-9.5 g/l; grade 3, 5.0-7.5 g/1; grade $3,<5.0 \mathrm{~g} / 1$. White blood cell: Grade $0 \geq 4.0 \times 10^{9} / 1$; grade $1,3.0-4.0 \times 10^{9} / 1$; grade 2, 2.0-3.0 $\times 10^{9} / 1$; grade $3,1.0-2.0 \times 10^{9} / 1$; grade $4,<1.0 \times 10^{9} / 1$. Platelet: Grade $0 \geq 100.0 \times 10^{9} / 1$; grade $1,75.0-100.0 \times 10^{9} / 1$; grade $2,50.0-75.0 \times 10^{9} / 1$; grade $3,25.0-75.0 \times 10^{9} / 1$; grade 4 , $<25.0 \times 10^{9} / 1$.

Follow-up. Follow-up was performed every 3 months among those with a survival of $<2$ years and every 6 months for those with a survival $>2$ years, according to the National Comprehensive Cancer Network guidelines (17). Data collection in the follow-up included case history, physical examinations, laboratory tests, electrocardiogram, abdominal ultrasound examination, and a barium esophagogram and thoracic CT scan. The OS time, PFS time and local control (LC; no recurrence in the primary lesion and local lymph node metastasis after radiotherapy) were used to evaluate the prognosis.

Statistical analysis. The primary endpoint was PFS and the secondary endpoints were the OS and LC. SPSS 19.0 software 
Table I. Comparison of the characteristics of patients with esophageal cancer in the control and test groups.

\begin{tabular}{|c|c|c|c|c|}
\hline Characteristic & $\mathrm{n}$ & Control group $(n=25)$ & Test group $(n=26)$ & P-value \\
\hline Sex & & & & $0.499^{\mathrm{a}}$ \\
\hline Male & 40 & 21 & 19 & \\
\hline Female & 11 & 4 & 7 & \\
\hline Age, years & & & & $0.843^{\mathrm{b}}$ \\
\hline $41-59$ & 17 & 8 & 9 & \\
\hline $60-75$ & 34 & 17 & 17 & \\
\hline Site of tumor & & & & $0.318^{\mathrm{a}}$ \\
\hline Chest, upper & 10 & 7 & 3 & \\
\hline Chest, middle segment & 21 & 10 & 11 & \\
\hline Chest, lower & 20 & 8 & 12 & \\
\hline Type of cancer & & & & $0.977^{\mathrm{a}}$ \\
\hline Medullary type & 49 & 24 & 25 & \\
\hline Ulcer type & 2 & 1 & 1 & \\
\hline T stage & & & & $0.090^{\mathrm{a}}$ \\
\hline $\mathrm{T} 1$ & 2 & 2 & 0 & \\
\hline $\mathrm{T} 2$ & 7 & 2 & 5 & \\
\hline $\mathrm{T} 3$ & 36 & 20 & 16 & \\
\hline $\mathrm{T} 4$ & 6 & 1 & 5 & \\
\hline $\mathrm{N}$ stage & & & & $0.169^{\mathrm{a}}$ \\
\hline No & 11 & 6 & 5 & \\
\hline $\mathrm{N} 1$ & 36 & 19 & 17 & \\
\hline $\mathrm{N} 2$ & 4 & 0 & 4 & \\
\hline TNM stage & & & & $0.343^{\mathrm{a}}$ \\
\hline I & 6 & 3 & 3 & \\
\hline II & 39 & 21 & 18 & \\
\hline III & 6 & 1 & 5 & \\
\hline
\end{tabular}

${ }^{a}$ Analysis was performed with Fisher's exact test. ${ }^{b}$ Analysis was performed with $\chi^{2}$ test. TNM, Tumor-Node-Metastasis.

(IBM Corp., Armonk, NY, USA) was used for the data analysis. Measurement data are presented as the mean \pm standard deviation. $\chi^{2}$ test or Fisher's exact test were used for the comparison of grouped data. Mann-Whitney rank sum test was performed for the comparison of ranked data in two groups. Univariate analysis was performed using the Kaplan-Meier method and a log rank test. Multivariate analysis was performed using the Cox proportional hazards model. $\mathrm{P}<0.05$ was considered to indicate a statistically significant difference.

\section{Results}

Patient characteristics. A total of 3 patients in the present study were recommended to receive other treatment options due to the presence of distal metastasis during radiotherapy. One case terminated the radiotherapy due to radiation pneumonitis, one case was transferred to another department due to presence of gallstones and one case denied subsequent therapy due to a high fever. In total, 2 patients denied the four-cycle chemotherapy. In addition, 2 patients completed the study but were lost during follow-up. Therefore, a total of 51 cases were included in the analysis (test group, 26; control group, 25). The characteristics or these patients are presented in Table I. No statistical differences were identified in the characteristics at the baseline level between the test group and control group $(\mathrm{P}>0.05)$.

Side-effects evaluation. The patients demonstrated no significant cardiac side-effects following radiotherapy. No statistical differences were revealed in the radiotherapy-associated esophageal injury and lung injury between the two groups ( $\mathrm{P}>0.05$; Table II). The present study also evaluated chemotherapy-associated adverse events, including blood count, vomiting, liver function, peripheral nerve injury, constipation and muscular pain. The data indicated no statistical differences between the two groups for these factors ( $\mathrm{P}>0.05$; Table III). The major side-effects for thalidomide included hypersomnia $(n=3,5$ and 3 for mild, moderate and severe, respectively). No cardiovascular system issues, including deep vein thrombosis, hypotension and bradycardia, were recorded.

Treatment response. In the test group, 16 patients demonstrated a complete response (CR) and 8 exhibited a partial response 
Table II. Toxicity of radiotherapy in the lung and esophagus.

\begin{tabular}{lcccccc}
\hline & \multicolumn{3}{c}{ Esophageal injury, $\%$} & & \multicolumn{2}{c}{ Lung injury, \% } \\
\cline { 2 - 4 } Group & Grade 1 & Grade 2 & Grade 3 & & Grade 1 & Grade 2 \\
\hline Control (n=25) & 32.0 & 60.0 & 8.0 & 44.0 & 48.0 & Grade 3 \\
Test (n=26) & 42.3 & 46.2 & 11.5 & 53.8 & 34.6 & -0.481 \\
Z-value & & -0.485 & & & 0.631 \\
P-value & & 0.628 & & & \\
\hline
\end{tabular}

Analysis was performed with Mann-Whitney rank sum test.

Table III. Comparison of chemotherapy-associated toxicities.

\begin{tabular}{|c|c|c|c|c|c|c|c|c|}
\hline \multirow[b]{2}{*}{ Variable } & \multirow[b]{2}{*}{ Group } & \multicolumn{5}{|c|}{ Toxicities, $\%$} & \multirow[b]{2}{*}{ Z-value } & \multirow[b]{2}{*}{ P-value } \\
\hline & & Grade 0 & Grade 1 & Grade 2 & Grade 3 & Grade 4 & & \\
\hline \multirow[t]{2}{*}{ Hemoglobin } & Control $(n=25)$ & 76.0 & 24.0 & 0.0 & 0.0 & 0.0 & -0.237 & 0.813 \\
\hline & Test $(n=26)$ & 73.1 & 26.9 & 0.0 & 0.0 & 0.0 & & \\
\hline \multirow[t]{2}{*}{ White blood cell } & Control $(n=25)$ & 0.0 & 56.0 & 36.0 & 4.0 & 4.0 & -0.368 & 0.713 \\
\hline & Test $(n=26)$ & 0.0 & 61.5 & 30.8 & 3.8 & 3.8 & & \\
\hline \multirow[t]{2}{*}{ Platelet } & Control $(n=25)$ & 64.0 & 28.0 & 8.0 & 0.0 & 0.0 & -0.261 & 0.794 \\
\hline & Test $(n=26)$ & 69.2 & 19.2 & 11.5 & 0.0 & 0.0 & & \\
\hline \multirow[t]{2}{*}{ Nausea and vomiting } & Control $(n=25)$ & 48.0 & 40.0 & 12.0 & 0.0 & 0.0 & -0.738 & 0.461 \\
\hline & Test $(n=26)$ & 57.7 & 34.6 & 7.7 & 0.0 & 0.0 & & \\
\hline \multirow[t]{2}{*}{ Liver function } & Control $(n=25)$ & 96.0 & 4.0 & 0.0 & 0.0 & 0.0 & -0.555 & 0.579 \\
\hline & Test $(n=26)$ & 92.3 & 7.7 & 0.0 & 0.0 & 0.0 & & \\
\hline \multirow[t]{2}{*}{ Peripheral nerve disease } & Control $(n=25)$ & 56.0 & 44.0 & 0.0 & 0.0 & 0.0 & -0.153 & 0.878 \\
\hline & Test $(n=26)$ & 53.8 & 46.2 & 0.0 & 0.0 & 0.0 & & \\
\hline \multirow[t]{2}{*}{ Constipation } & Control $(n=25)$ & 44.0 & 40.0 & 12.0 & 4.0 & 0.0 & -0.041 & 0.968 \\
\hline & Test $(n=26)$ & 50.0 & 23.1 & 23.1 & 3.8 & 0.0 & & \\
\hline \multirow[t]{2}{*}{ Muscular pain } & Control $(n=25)$ & 72.0 & 28.0 & 0.0 & 0.0 & 0.0 & -1.466 & 0.143 \\
\hline & Test $(n=26)$ & 88.5 & 11.5 & 0.0 & 0.0 & 0.0 & & \\
\hline
\end{tabular}

Analysis was performed with Mann-Whitney rank sum test.

Table IV. Comparison of the total effectiveness rates.

\begin{tabular}{llcc}
\hline Group & CR, \% & PR, \% & SD, \% \\
\hline Test (n=26) & 61.5 & 30.8 & 7.7 \\
Control (n=25) & 60.0 & 32.0 & 8.0 \\
Z-value & & -0.109 & \\
P-value & & 0.913 & \\
\hline
\end{tabular}

Analysis was performed with Mann-Whitney rank sum test; CR, complete response; $\mathrm{PR}$, partial response; SD, stable disease.

(PR). In the control group, 15 demonstrated a CR and 8 exhibited a PR. The total treatment effective rate demonstrated no statistical differences between the two groups (92.3 vs. $92.0 \%$; $\mathrm{P}>0.05$; Table IV).
Prognosis analysis. In all cases, the 1-year and 3-year OS rates were 70.6 and $22.5 \%$, respectively. The 1 -year and 3 -year PFS rates were 56.9 and $22.0 \%$, respectively. In addition, the 1-year and 3-year LC rates were 81.0 and $52.6 \%$, respectively. The comparison of these data between the test and control group was shown in Table V. For the 9 patients with cancer recurrence, the median time to recurrence was 7.5 months [95\% confidence interval (CI), 1.3-13.7 months], and the median OS and PFS times were 20.6 months (95\% CI, 15.1-26.2 months) and 17.0 months (95\% CI, 8.8-25.2 months), respectively. The median OS time in the test group was 36.8 months (95\% CI, 14.0-59.5 months), while that for the control group was 17.0 months $(95 \%$ CI, 8.8-25.8 months), respectively ( $\mathrm{P}=0.104$; Fig. 1A). The median PFS time in the test group was 36.2 months (95\% CI, 13.2-58.8 months), while that in the control group was 13.9 months (95\% CI, 3.7-24.0 months), respectively $(\mathrm{P}=0.063$; Fig. 1B). 

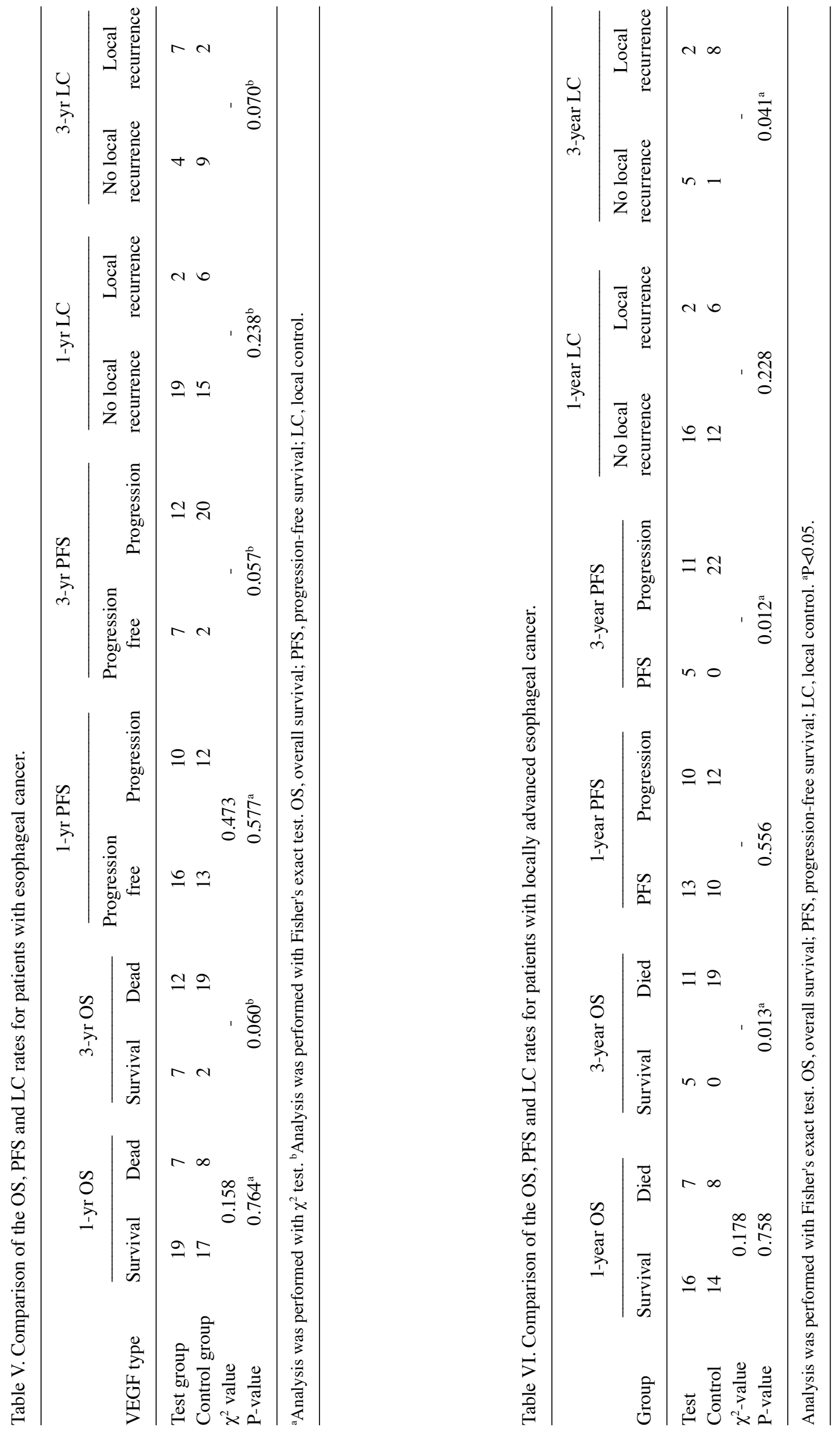

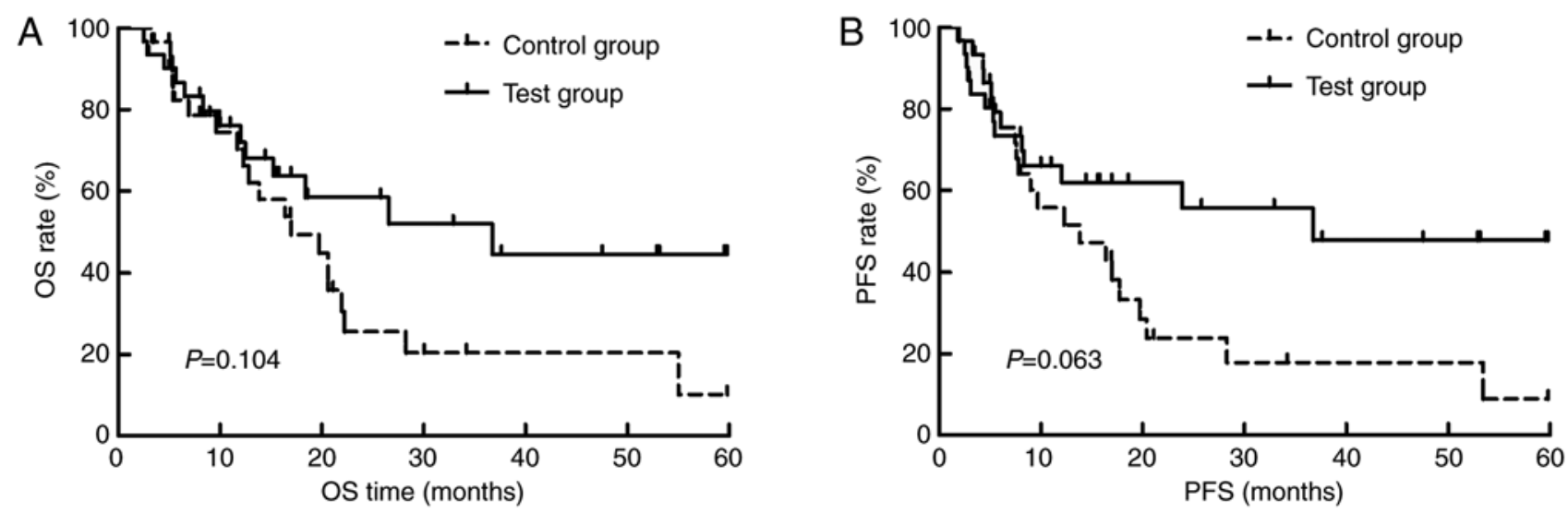

Figure 1. OS and PFS time analysis of all patients with EC. Comparison of the (A) OS and (B) PFS time of patients with EC in the test and control groups. OS, overall survival; PFS, progression-free survival; EC, esophageal cancer.
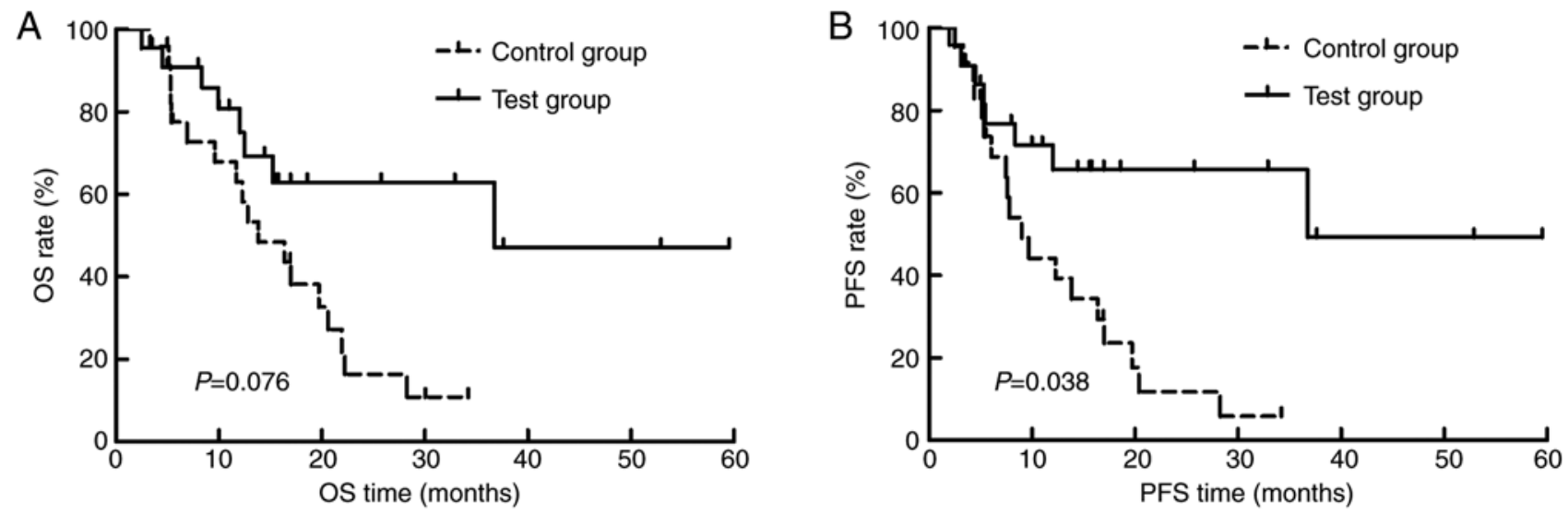

Figure 2. OS and PFS time analysis of patients with locally advanced EC. Comparison of the (A) OS and (B) PFS time of patients with locally advanced EC in the test and control groups. OS, overall survival; PFS, progression-free survival; EC, esophageal cancer.

Prognosis analysis in locally advanced patients. Among the 61 cases, 55 were confirmed with locally advanced EC (test group, $n=23$; control group, $n=22$ ). Locally advanced EC referred to primary lesions of $\geq \mathrm{T} 3$ or positive for lymph nodes. Table VI summarizes the OS, PFS and LC rates in the test and control groups of patients with locally advanced EC. The 3 -year OS, PFS and LC rates in the test group were higher than that of the control. The median OS times in the test and control groups were 36.8 months (95\% CI, 21.2-46.3 months) and 16.4 months (95\% CI, 10.5-22.3 months), respectively ( $\mathrm{P}=0.076$; Fig. 2A). The median PFS time was 36.8 months (95\% CI, 20.2-47.3 months) in the test group, while that for the control group was 9.7 months (95\% CI, 3.6-15.9 months), respectively ( $\mathrm{P}=0.038$; Fig. 2B).

Effects of thalidomide on the change of serum VEGF. The changes of VEGF level were classified as decrease, stable or increase compared with the level during radiotherapy. Compared with the control group, statistical differences were identified in the changes of VEGF between the test group and control group $(\mathrm{P}<0.05$; Table VII).

Associations between VEGF changes and prognosis. Subsequently, the associations between VEGF changes and the OS, PFS and LC rates for patients with EC were evaluated. Patients with increased VEGF following radiotherapy all died within 1 year. A significant increase was identified in the 1-year OS, 1-year PFS, 1-year LC and 3-year LC rates for patients with stable or decreased VEGF compared with those with an increase of VEGF ( $\mathrm{P}<0.05$; Table VIII).

Analysis of prognostic factors. Univariate analysis revealed that the OS time was not associated with sex, age, site and type of tumors, KPS, pre-treatment cancer length, tumor diameter, $\mathrm{N}$ stage, radiotherapy dose, administration of thalidomide or the presence of residual cancer after radiotherapy (Table IX). However, $\mathrm{T}$ stage $(\mathrm{P}=0.028)$ and TNM stage $(\mathrm{P}=0.024)$ were identified to be significantly associated with OS time (Table IX). Cox multivariate analysis demonstrated that compared with stage I patients, the risk of mortality increased in stage II [relative risk (RR), 5.613; 95\% CI, 1.161-27.127; $\mathrm{P}<0.05$ ] and III patients (RR, 8.097; 95\% CI, 1.312-49.972; $\mathrm{P}<0.05)$ after adjusting for age, sex and administration of thalidomide (Table X). For the PFS time, univariate analysis revealed age, sex, site and type of tumor, KPS, pre-treatment tumor length, tumor diameter, $\mathrm{N}$ stage, dose of radiotherapy and administration of thalidomide were not significant associated. By contrast, the presence of residual cancer cell after radiotherapy 
Table VII. Changes of VEGF in control and test groups post-radiotherapy compared with during radiotherapy.

\begin{tabular}{lccc}
\hline Group & $\begin{array}{c}\text { Decreased } \\
\text { VEGF, \% }\end{array}$ & $\begin{array}{c}\text { Stable } \\
\text { VEGF, \% }\end{array}$ & $\begin{array}{r}\text { Elevated } \\
\text { VEGF, \% }\end{array}$ \\
\hline Test (n=26) & 53.8 & 38.5 & 7.7 \\
Control (n=25) & 16.0 & 64.0 & 20.0 \\
Z-value & & -2.745 & \\
P-value & & $0.006^{\mathrm{a}}$ & \\
\hline
\end{tabular}

Analysis was performed with Mann-Whitney rank sum test; VEGF, vascular endothelial growth factor. ${ }^{\mathrm{a}} \mathrm{P}<0.05$.

$(\mathrm{P}=0.006)$, $\mathrm{T}$ stage $(\mathrm{P}=0.025)$ and TNM stage $(\mathrm{P}=0.019)$ were identified to be associated with PFS time (Table XI). Cox multivariate analysis demonstrated that compared with stage I patients, the risk of disease progression increased in stage II (RR, 4.190; 95\% CI, 0.630-27.853; $\mathrm{P}<0.05$ ) and III patients (RR, 5.693; 95\% CI, 1.117-29.025; $\mathrm{P}<0.05)$ after adjusting for age, sex and administration of thalidomide (Table XII). Compared with those with $\mathrm{CR}$ following radiotherapy, patients with the presence of residue EC cells demonstrated a significant increase in disease progression after radiotherapy (RR, 1.910; 95\% CI, 1.246-2.930; P<0.05; Table XII).

\section{Discussion}

Surgery is not recommended for the majority of patients who present with advanced EC upon diagnosis. In a previous study, CRT demonstrated a comparable efficiency to surgery for patients with EC (18). Therefore, concurrent CRT is recommended by the National Cancer Institute Network as a standard treatment option for EC (18). The present study compared the treatment efficiency, prognosis and side-effects between CRT and a combination of CRT and thalidomide.

VEGF, a mitogen-activated factor secreted by vascular endothelial cells, serves important roles in several biological processes, including differentiation of endothelial cells, elevation of capillary permeability, endothelial cell migration and angiogenesis (19). VEGF has been identified to be expressed in vascular endothelial cells, esophageal endothelial cells and mononuclear macrophages. In addition, it is expressed in several types of malignant cell including cancer cells and tumor vascular endothelial cell. Furthermore, it has been detected in serum and exudate (20). Generally, the expression of VEGF in the normal tissues was relatively low, as it could only maintain vascular density and basic osmosis, which contributed to the nutrition delivery (21). The expression of VEGF in cancer tissues is markedly higher compared with normal tissues as the growth of cancer depends on the transport of nutrients mediated by blood vessels (11). VEGF has been reported to serve a pivotal role in vascularization (11). In addition, VEGF is considered as an independent prognosis factor and is closely associated with the recurrence and metastasis of cancer (22). Serum VEGF has been reported to be closely associated with tumor load, infiltration and lymph node metastasis. The expression of VEGF was identified to

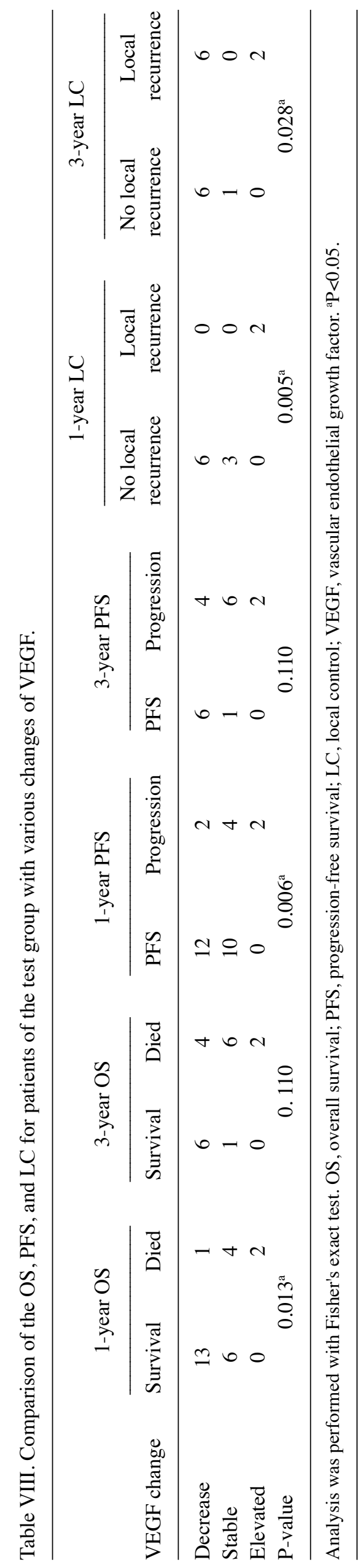


Table IX. Univariate analysis of the overall survival rate of patients with esophageal cancer.

\begin{tabular}{lcccccc}
\hline Variable & $\beta$-value & Standard error & Wald-value & RR & $95 \%$ CI & P-value \\
\hline Sex & 0.261 & 0.429 & 0.371 & 1.298 & $0.560-3.009$ & 0.543 \\
Age & -0.025 & 0.027 & 0.897 & 0.975 & $0.926-1.027$ & 0.343 \\
KPS & 0.038 & 0.038 & 0.991 & 1.039 & $0.964-1.121$ & 0.320 \\
Lesion position & -0.132 & 0.248 & 0.286 & 0.876 & $0.539-1.423$ & 0.593 \\
Lesion type & 0.421 & 0.749 & 0.315 & 1.523 & $0.351-6.606$ & 0.574 \\
T stage & 0.710 & 0.324 & 4.808 & 2.033 & $1.078-3.834$ & $0.028^{\mathrm{a}}$ \\
N stage & 0.670 & 0.345 & 3.779 & 1.955 & $0.994-3.844$ & 0.052 \\
TNM stage & 0.634 & 0.282 & 5.066 & 1.885 & $1.085-3.275$ & $0.024^{\mathrm{a}}$ \\
Cancer length before treatment & 0.134 & 0.088 & 2.358 & 1.144 & $0.964-1.358$ & 0.125 \\
Lesion diameter & 0.033 & 0.197 & 0.027 & 1.033 & $0.702-1.520$ & 0.868 \\
Residual cancer cells after radiotherapy & 0.365 & 0.194 & 3.541 & 1.441 & $0.985-2.109$ & 0.060 \\
Radiotherapy dose & -0.058 & 0.035 & 2.774 & 0.944 & $0.882-1.010$ & 0.096 \\
Thalidomide & -0.575 & 0.359 & 2.568 & 0.562 & $0.278-1.137$ & 0.109
\end{tabular}

KPS, Karnofsky performance score; TNM, Tumor-Node-Metastasis; CI, confidence interval; RR, relative risk. ${ }^{\text {a }}<0.05$.

Table X. Cox multivariate analysis of the overall survival rate of patients with esophageal cancer.

\begin{tabular}{lccc}
\hline Variable & RR & $95 \% \mathrm{CI}$ & P-value \\
\hline Sex & & & \\
$\quad$ Male & 1.000 & - & \\
Female & 0.745 & $0.311-1.785$ & 0.509 \\
Age & 1.006 & $0.952-1.063$ & 0.838 \\
TNM stage & & & \\
Stage I & 1.000 & - & $0.032^{\mathrm{a}}$ \\
Stage II & 5.613 & $1.161-27.127$ & $0.024^{\mathrm{a}}$ \\
Stage III & 8.097 & $1.312-49.972$ & \\
Thalidomide & & & 0.088 \\
No & 1.000 & - & \\
Yes & 0.523 & $0.248-1.101$ & \\
\hline
\end{tabular}

TNM, Tumor-Node-Metastasis; CI, confidence interval; RR, relative risk. ${ }^{\mathrm{a}} \mathrm{P}<0.05$.

be significantly higher in patients with a large tumor volume and lymph node metastasis compared with those with a small tumor volume and the absence of lymph node metastasis (22). Our previous study (19) demonstrated that VEGF level is associated with the sensitivity to radiotherapy and prognosis of chemo-radiotherapy, and the treatment efficiency in patients with downregulated VEGF was higher compared with those with an increased level of VEGF. In patients with EC with a satisfactory prognosis, the VEGF level during radiotherapy was demonstrated to be decreased compared with the baseline level. The elevation of VEGF induced by radiotherapy may be associated with the self-protection of cancer cells to encounter the toxicity of radiotherapy.
Thalidomide was initially utilized for the management of lepra reactions and was then demonstrated to be effective for attenuating vomiting and nausea in early gestation. However, the application of thalidomide is limited as it causes congenital defects in neonates $(10,12)$. Since then, studies involving thalidomide predominantly focused on the immune system, anti-inflammation and anti-angiogenesis. In 1999, Singhal et al (23) reported that thalidomide exhibited antitumor activity in refractory multiple myeloma with a CR rate of $32 \%$. Subsequently, thalidomide has been demonstrated to be effective for the treatment of various types pf hematologic neoplasm (24-26). Thalidomide has been regarded as a treatment option for solid tumors (27) and the majority of studies have been focused on anti-angiogenesis, chemotherapy sensitivity and attenuation of the chemotherapy-associated side-effects $(28,29)$. To the best of our knowledge, no previous studies have investigated the efficiency of thalidomide for concomitant therapy with CRT. Previously, thalidomide has been reported to increase the radiotherapy sensitivity of EC cells (30). Our previous study (22) demonstrated that a combination of thalidomide and radiotherapy could trigger a downregulation of VEGF, specifically patients receiving thalidomide demonstrated satisfactory tolerance to radiotherapy.

In the present study, patients with EC with an elevated VEGF level were randomly divided into the test group and control group to investigate the effects of thalidomide on VEGF and prognosis. The data revealed that the short-term treatment efficiency was similar between the two groups, and the 1-year and 3-year OS, PFS and LC rates, and median OS and PFS times demonstrated no statistical differences. The stratified analysis revealed that the patients with locally advanced EC in the test group exhibited significantly higher 3-year OS, PFS and LC rates and median PFS time compared with the control group, which indicates that a combination of thalidomide and chemo-radiotherapy contributes to the prognosis of patients 
Table XI. Univariate analysis of the progression-free survival rate of patients with esophageal cancer.

\begin{tabular}{|c|c|c|c|c|c|c|}
\hline Variable & $\beta$-value & Standard error & Wald-value & RR & $95 \% \mathrm{CI}$ & P-value \\
\hline Sex & -0.312 & 0.427 & 0.534 & 0.732 & $0.317-1.691$ & 0.465 \\
\hline Age & -0.042 & 0.026 & 2.580 & 0.959 & 0.911-1.009 & 0.108 \\
\hline KPS & 0.048 & 0.038 & 1.572 & 1.049 & $0.973-1.131$ & 0.210 \\
\hline Lesion position & -0.069 & 0.242 & 0.082 & 0.933 & $0.581-1.499$ & 0.774 \\
\hline Lesion type & 0.184 & 0.742 & 0.061 & 1.202 & $0.281-5.146$ & 0.804 \\
\hline T stage & 0.727 & 0.325 & 5.022 & 2.070 & $1.096-3.910$ & $0.025^{\mathrm{a}}$ \\
\hline $\mathrm{N}$ stage & 0.706 & 0.360 & 3.849 & 2.027 & $1.001-4.105$ & 0.050 \\
\hline TNM stage & 0.658 & 0.282 & 5.468 & 1.932 & $1.112-3.354$ & $0.019^{\mathrm{a}}$ \\
\hline Cancer length before treatment & 0.155 & 0.090 & 2.997 & 1.168 & $0.980-1.393$ & 0.083 \\
\hline Lesion diameter & 0.198 & 0.195 & 1.028 & 1.219 & $0.831-1.787$ & 0.311 \\
\hline Residual cancer cells after radiotherapy & 0.535 & 0.194 & 7.627 & 1.707 & $1.168-2.495$ & $0.006^{\mathrm{a}}$ \\
\hline Radiotherapy dose & -.045 & 0.036 & 1.608 & 0.956 & $0.891-1.025$ & 0.205 \\
\hline Thalidomide & -.652 & 0.357 & 3.340 & 0.521 & $0.259-1.048$ & 0.068 \\
\hline
\end{tabular}

KPS, Karnofsky performance score; TNM, Tumor-Node-Metastasis; CI, confidence interval; RR, relative risk. ${ }^{a} \mathrm{P}<0.05$.

Table XII. Cox multivariate analysis of the progression-free survival rate of patients with esophageal cancer.

\begin{tabular}{lccc}
\hline Variable & RR & $95 \%$ CI & P-value \\
\hline Sex & & & \\
$\quad$ Male & 1.000 & - & \\
$\quad$ Female & 0.853 & $0.346-2.108$ & 0.731 \\
Age & 0.962 & $0.909-1.018$ & 0.178 \\
TNM stage & & & \\
Stage I & 1.000 & - & \\
$\quad$ Stage II & 4.190 & $0.630-27.853$ & 0.138 \\
$\quad$ Stage III & 5.693 & $1.117-29.025$ & $0.036^{\mathrm{a}}$ \\
Thalidomide & & & \\
$\quad$ No & 1.000 & - & \\
$\quad \begin{array}{l}\text { Yes } \\
\text { Residual cancer cells }\end{array}$ & 1.910 & $1.246-2.930$ & $0.003^{\mathrm{a}}$ \\
after radiotherapy & & & \\
\hline
\end{tabular}

TNM, Tumor-Node-Metastasis; CI, confidence interval; RR, relative risk. ${ }^{\mathrm{a} P}<0.05$

with locally advanced EC with elevated VEGF. Compared with the control group, the post-treatment VEGF level demonstrated a significant decrease in the test group. This indicates that thalidomide contributes to the decrease of serum VEGF level in patients with EC with elevated VEGF during the concurrent CRT. Furthermore, the OS, PFS and LC rates for patients with decreased VEGF following administration of thalidomide were higher compared with those with increased VEGF, which indicates that the benefits of thalidomide may be associated with the inhibition of VEGF in cancer tissues. Additionally, multivariate analysis demonstrated that TNM stage was significantly associated with OS time, while the presence of residual cancer cells and TNM stage were significantly associated with the PFS time following radiotherapy.

There were certain limitations of the present study. Firstly, the sample size was too small. To improve this, more data from a larger number of patients should be included in future studies. In addition, no significant differences were identified in the 1-year OS and PFS rates for the patients with locally advanced EC between the two groups. This may be due to the small sample size. Furthermore, the present study only focused on the side-effects, treatment efficiency and prognosis of thalidomide combined with CRT. Therefore, potential mechanisms should be investigated in future studies.

In conclusion, the current study demonstrated that thalidomide contributes to an improvement of prognosis for patients with locally advanced EC with elevated serum VEGF during radiotherapy. Furthermore, the toxicities observed were tolerable. It was concluded that the benefits of thalidomide in clinical practice may be associated with the inhibition of VEGF in cancer cells.

\section{Acknowledgements}

Not applicable.

\section{Funding}

This study was supported by the National Natural Science Foundation of China (grant no. 11705095), the Key Science \& Technology Program of Changzhou Municipal Commission of Health and family planning (grant no. ZD201710), the Changzhou Scientific and Technological Support Social Development Project (grant no. CE20165024) and the Changzhou Municipal Science and Technology Bureau Basic Research Project (grant no. CJ20159050). 


\section{Availability of data and materials}

The raw data were available upon appropriate requests.

\section{Authors' contributions}

JW was responsible for data collection and analysis, as well as manuscript writing. JY, JLW, XN, ZS and WS were responsible for data collection and analysis. SS and YL designed the study and revised the manuscript.

\section{Ethics approval and consent to participate}

Written informed consent was obtained from each patient. The study protocols were approved by the Ethics Committee of the Second People's Hospital of Changzhou Affiliated to Nanjing Medical University (Nanjing, China). This clinical trial was registered in the United States Trial Registry (ID: NCT01551641).

\section{Patient consent for publication}

Not applicable.

\section{Competing interests}

The authors declare that they have no competing interests.

\section{References}

1. Siegel R, Desantis C and Jemal A: Colorectal cancer statistics, 2014. CA Cancer J Clin 64: 104-117, 2014.

2. DaVee T, Ajani JA and Lee JH: Is endoscopic ultrasound examination necessary in the management of esophageal cancer? World J Gastroenterol 23: 751-762, 2017.

3. Cooper JS, Guo MD, Herskovic A, Macdonald JS, Martenson JA Jr, Al-Sarraf M, Byhardt R, Russell AH, Beitler JJ, Spencer S, et al Chemoradiotherapy of locally advanced esophageal cancer: Long-term follow-up of a prospective randomized trial (RTOG 85-01). Radiation Therapy Oncology Group. JAMA 281: 1623-1627, 1999.

4. Zhao KL, Liu G, Jiang GL, Wang Y, Zhong LJ, Wang Y, Yao WQ, Guo XM, Wu GD, Zhu LX and Shi XH: Association of haemoglobin level with morbidity and mortality of patients with locally advanced oesophageal carcinoma undergoing radiotherapy-a secondary analysis of three consecutive clinical phase III trials. Clin Oncol (R Coll Radiol) 18: 621-627, 2006.

5. Maouris PG and Hirsch PJ: Pregnancy in women with thalidomideinduced disabilities. Case report and a questionnaire study. Br J Obstet Gynaecol 95: 717-719, 1988.

6. Zhou S, Wang F, Hsieh TC, Wu JM and Wu E: Thalidomide-a notorious sedative to a wonder anticancer drug. Curr Med Chem 20: 4102-4108, 2013.

7. Rades D, Golke H, Schild SE and Kilic E: Impact of VEGF and VEGF receptor 1 (FLT1) expression on the prognosis of stage III esophageal cancer patients after radiochemotherapy. Strahlenther Onkol 184: 416-420, 2008.

8. Yoon MS, Nam TK, Lee JS, Cho SH, Song JY, Ahn SJ, Chung IJ, Jeong JU, Chung WK and Nah BS: VEGF as a predictor for response to definitive chemoradiotherapy and COX-2 as a prognosticator for survival in esophageal squamous cell carcinoma J Korean Med Sci 26: 513-520, 2011.

9. Yu J, Liu F, Sun M, Sun Z and Sun S: Enhancement of radiosensitivity and the potential mechanism on human esophageal carcinoma cells by tetrandrine. Cancer Biother Radiopharm 26: 437-442, 2011.

10. Yu J, Liu F, Sun Z, Sun M and Sun S: The enhancement of radiosensitivity in human esophageal carcinoma cells by thalidomide and its potential mechanism. Cancer Biother Radiopharm 26 : 219-227, 2011
11. Wang J, Yu JP, Wang JL, Ni XC, Sun ZQ, Sun W, Nie B, Jiang JT, Sun SP and Wu CP: Pathologic response and changes of serum VEGF during chemoradiotherapy may predict prognosis in non-surgical patients with esophageal carcinoma. Zhonghua Zhong Liu Za Zhi 38: 589-595, 2016 (In Chinese).

12. Yu JP, Sun SP, Sun ZQ, Ni XC, Wang J, Li Y, Hu LJ and Li DQ: Clinical trial of thalidomide combined with radiotherapy in patients with esophageal cancer. World J Gastroenterol 20: 5098-5103, 2014.

13. Milstein JM, Cohen ME and Sinks LF: The influence and reliability of neurologic assessment and Karnofsky performance score on prognosis. Cancer 56 (7 Suppl): S1834-S1836, 1985.

14. Strong VE, D'Amico TA, Kleinberg L and Ajani J: Impact of the 7th Edition AJCC staging classification on the NCCN clinical practice guidelines in oncology for gastric and esophageal cancers. J Natl Compr Canc Netw 11: 60-66, 2013.

15. Coche E: Recist and beyond. JBR-BTR 96: 167-171, 2013.

16. Zhang TR, Zhao T, Xu X, Gu XW and Pan YK: Efficacy and side-effects of docetaxel combined with cisplatin on the treatment of local advanced esophageal cancer with concomitant radiation therapy. Zhonghua Zhong Liu Za Zhi 32: 791-794, 2010 (In Chinese).

17. National Comprehensive Cancer Net work. NCCN, 2016 [EB/OL] https:// www.nccn.org/. Accessed August 20, 2016.

18. Crehange G, Maingon P, Peignaux K, N'guyen TD, Mirabel X, Marchal C, Verrelle P, Roullet B, Bonnetain F and Bedenne L; Federation Francophone de Cancerologie Digestive 9102: Phase III trial of protracted compared with split-course chemoradiation for esophageal carcinoma: Federation francophone de cancerologie digestive 9102. J Clin Oncol 25: 4895-4901, 2007.

19. Yu JP, Lu WB, Wang JL, Ni XC, Wang J, Sun ZQ and Sun SP: Pathologic response during chemo-radiotherapy and variation of serum VEGF levels could predict effects of chemo-radiotherapy in patients with esophageal cancer. Asian Pac J Cancer Prev 16: 1111-1116, 2015.

20. Lu JJ, Ma J, Miao R, Gu Y and Zhong FT: Expression of vascular endothelial growth factor D in human esophageal squamous cell carcinoma tissue and its significance. Zhonghua Wei Chang Wai Ke Za Zhi 16: 1191-1194, 2013 (In Chinese).

21. Bedoya F, Meneu JC, Macías MI, Moreno A, Enríquez-De-Salamanca R, Gonzalez EM and Vegh I: Mutation in CNR 1 gene and VEGF expression in esophageal cancer. Tumori 95: 68-75, 2009.

22. Srivastava VK, Gara RK, Rastogi N, Mishra DP, Ahmed MK, Gupta S, Goel MM and Bhatt ML: Serum vascular endothelial growth factor-A (VEGF-A) as a biomarker in squamous cell carcinoma of head and neck patients undergoing chemoradiotherapy. Asian Pac J Cancer Prev 15: 3261-3265, 2014.

23. Singhal S, Mehta J, Desikan R, Ayers D, Roberson P, Eddlemon P, Munshi N, Anaissie E, Wilson C, Dhodapkar M, et al: Antitumor activity of thalidomide in refractory multiple myeloma. N Engl J Med 341: 1565-1571, 1999.

24. Steins MB, Bieker R, Padró T, Kessler T, Kienast J, Berdel WE and Mesters RM: Thalidomide for the treatment of acute myeloid leukemia. Leuk Lymphoma 44: 1489-1493, 2003.

25. Kay NE, Shanafelt TD, Call TG, Wu W and Laplant BR: N9986: A phase II trial of thalidomide in patients with relapsed chronic lymphocytic leukemia. Leuk Lymphoma 50: 588-592, 2009.

26. Townsend W, Johnson RJ, Pottinger BT, Counsell N, Smith P, Chadwick H, Evans K, Wickham C and Rudin CE; UK NCRI Lymphoma Clinical Studies Group: A phase II clinical trial of fludarabine and cyclophosphamide followed by thalidomide for angioimmunoblastic T-cell lymphoma. An NCRI clinical trial. CRUK number C17050/A5320. Leuk Lymphoma 57: 2232-2234, 2016.

27. Kumar S, Witzig TE and Rajkumar SV: Thalidomid: Current role in the treatment of non-plasma cell malignancies. J Clin Oncol 22: 2477-2488, 2004.

28. Rehman W, Arfons LM and Lazarus HM: The rise, fall and subsequent triumph of thalidomide: Lessons learned in drug development. Ther Adv Hematol 2: 291-308, 2011.

29. Sauer H, Günther J, Hescheler J and Wartenberg M: Thalidomide inhibits angiogenesis in embryoid bodies by the generation of hydroxyl radicals. Am J Pathol 156: 151-158, 2000.

30. Goel S, Wong AH and Jain RK: Vascular normalization as a therapeutic strategy for malignant and nonmalignant disease. Cold Spring Harb Perspect Med 2: a006486, 2012. 